\title{
Tolerance to Fusarium Root Rot and the Changes in Free Amino Acid Contents in Mycorrhizal Asparagus Plants
}

\author{
Tomohiro Okada \\ The United Graduate School of Agricultural Science, Gifu University, 1-1 \\ Yanagido, Gifu 501-1193, Japan
}

Yoh-ichi Matsubara ${ }^{1}$

Faculty of Applied Biological Sciences, Gifu University, 1-1 Yanagido, Gifu 501-1193, Japan

Additional index words. arbuscular mycorrhizal fungus, arginine, asparagus decline, Fusarium oxysporum f. sp. asparagi, GABA, growth promotion, symbiosis

\begin{abstract}
Tolerance to fusarium root rot and the changes in free amino acid contents in mycorrhizal asparagus (Asparagus officinalis L., cv. Welcome) plants were investigated. Sixteen weeks after inoculation of arbuscular mycorrhizal fungus (AMF; Glomus sp. R10), mycorrhizal plants showed higher dry weight of ferns and roots than nonmycorrhizal plants, and AMF colonization level in a root system reached up to $73.3 \%$. Ten weeks after Fusarium oxysporum f. sp. asparagi (Foa; MAFF305556, SUF1226) inoculation, disease incidence and the severity of symptoms were eased and disease indices were low as less than 20 in mycorrhizal plants compared with non-AMF plants in the both isolates. As for the changes in free amino acid, total free amino acid contents in ferns and roots were higher in AMF plants than non-AMF plants 16 weeks after AMF inoculation. In this case, eight constituents of amino acids in ferns and 16 in roots increased in AMF plants; in particular, arginine and gamma-aminobutyric acid (GABA) showed considerable increase in both ferns and roots in AMF plants. In the Foa culture by Czapec-Dox medium in vitro, suppression of Foa propagation was recognized by the addition $(0.1,1 \%, w / v)$ of arginine and GABA. From these findings, plant growth enhancement and tolerance to fusarium root rot occurred in mycorrhizal asparagus plants, and the disease tolerance was supposed to be associated with the symbiosisspecific increase in free amino acids.
\end{abstract}

Asparagus decline is a serious and increasing threat in asparagus producing regions over the world (Hamel et al., 2005; Knaflewski et al., 2008; Reid et al., 2001; Wong and Jeffries, 2006). It is supposed to be caused by the contribution of both biotic factors (Knaflewski et al., 2008; Wong and Jeffries, 2006) and abiotic factors (Lake et al., 1993; Miller et al., 1991; Yong, 1984). As biotic factors, the most common phenomenon is fusarium crown and root rot caused by Foa, Fusarium proliferatum (Fp), and Fusarium redolens etc. (Knaflewski et al., 2008; Reid et al., 2002; Wong and Jeffries, 2006). In Japan, Nahiyan et al. (2011) demonstrated that Foa and $\mathrm{Fp}$ are dominant fusarium species in asparagus decline fields. However, the diseases are still difficult to control because no resistant cultivar or disinfesting method has been developed. On the other hand, biological

Received for publication 1 Feb. 2012. Accepted for publication 6 Apr. 2012.

This study was supported by Grants-in-Aid for Scientific Research (No. 21580029), Japan Society for the Promotion of Science (JSPS).

${ }^{1}$ To whom reprint requests should be addressed; e-mailymatsu@gifu-u.ac.jp. control of fusarium disease was tried by the inoculation with non-pathogenic isolates of the fusarium species (Blok et al., 1997; Elmer, 2004). However, the method is not enough to control the diseases and has no growthpromoting effect.

Arbuscular mycorrhizal fungi are ubiquitous soil inhabitants and form a symbiotic relationship with roots of most of the terrestrial plants. AMF promotes host plant growth by enhancing phosphorus uptake through symbiosis (Marschner and Dell, 1994) and hence an alternative to high inputs of fertilizers and pesticides in sustainable crop production systems. Previously, we reported tolerance to fusarium root rot in mycorrhizal asparagus (cv. Mary Washington 500W) plants (Matsubara et al., 2003); however, many points remain unclear about the mechanisms of disease tolerance in mycorrhizal asparagus plants.

As for the changes in amino acid constituents related to disease tolerance in mycorrhizal plants, Baltruschat and Schonbeck (1975) demonstrated that the propagation of Thielaviopsis basicola was inhibited by the increase of arginine and citrulline in mycorrhizal tobacco plants. In addition, some reports mentioned that the free amino acid level in plants changes through AMF colonization. Sood (2003) and Fattah and Mohamedin (2000) reported that increases in the contents of free amino acids occurred in mycorrhizal tomato and sorghum plants, respectively. On the other hand, Rolin et al. (2001) reported that AMF colonization decreased total amino acid levels in mycorrhizal leek plants. However, it has been unclear how the contents of free amino acid change through symbiosis with $\mathrm{AMF}$ in asparagus plants and how the changes are associated with disease tolerance.

In this study, tolerance to fusarium root rot and the changes in free amino acid contents in mycorrhizal asparagus plants were investigated to clarify the relationship between free amino acid and disease tolerance.

\section{Materials and Methods}

Arbuscular mycorrhizal fungi inoculation and cultivation condition. Seeds of asparagus (Asparagus officinalis L., cv. Welcome) were sown in commercial soil (autoclaved at 1.2 $\mathrm{kg} \cdot \mathrm{cm}^{-2}$ and $121^{\circ} \mathrm{C}$ for $1 \mathrm{~h}$ ) in plastic container $(43 \times 27 \times 17 \mathrm{~cm})$. During the time of seed sowing, plant holes were made and each hole contains $3 \mathrm{~g} /$ plant commercial AMF (Glomus sp. R10) inoculum supplied by Idemitsukosan Co. Ltd., Tokyo, Japan. Then, seeds were sown onto the inoculum, finally covered with soil, and administered by mixed fertilizer $(13 \mathrm{~N}-11 \mathrm{P}-13 \mathrm{~K}, 0.5 \mathrm{~g}$ per plant). Forty plants per plot with three replications were irrigated as regularly and grown in a greenhouse.

Inoculation of Fusarium oxysporum $\mathrm{f}$. $\mathrm{sp}$. asparagi. Two isolates of Foa (MAFF305556 and SUF1226) were grown on potato-dextrose agar media. The conidia were harvested in potato sucrose liquid media and incubated at $25{ }^{\circ} \mathrm{C}$ in the dark for $7 \mathrm{~d}$. The conidial suspension was sieved and the concentrations adjusted to $10^{6}$ conidia/mL. Sixteen weeks after AMF inoculation, each plant was inoculated by $50 \mathrm{~mL}$ of the conidial suspension onto the roots.

Estimation of symptoms of fusarium root rot. Ten weeks after inoculation of Foa, the symptoms of fusarium root rot were rated to 6 degrees as 0 , no symptom; 1 , less than $20 \%$; $20 \%$ to $40 \% ; 3,40 \%$ to $60 \%$; $4,60 \%$ to $80 \%$; $5,80 \%$ to $100 \%$. In addition, the disease index was calculated by the following formula:

$$
\begin{aligned}
& \text { Disease index }= \\
& \sum \text { (number of plants } \\
& \times \text { number of degree } \\
& \text { in symptoms) } \\
& \hline \text { Total number of plants } \\
& \times 5 \text { (maximum degree } \\
& \text { in symptom) }
\end{aligned}
$$

Evaluation of arbuscular mycorrhizal fungi colonization level. Sixteen weeks after AMF inoculation, roots of asparagus were preserved with $70 \%$ ethanol and stained according to Phillips and Hayman (1970). The rate of AMF colonization in $1-\mathrm{cm}$ 

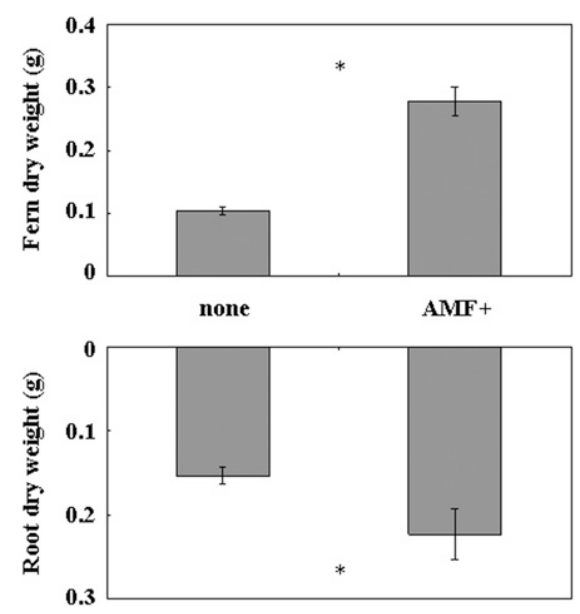

Fig. 1. Dry weight of asparagus plants 16 weeks after AMF inoculation. None = none-AMFinoculated; $\mathrm{AMF}+=$ Glomus sp. R10. Bars represent SES $(\mathrm{n}=10)$. * significantly different between non-AMF and AMF plants $(t$ test, $P \leq$ $0.05) ; \mathrm{NS}=$ non-significant. AMF $=$ arbuscular mycorrhizal fungus.

segments of lateral roots (abbreviated RFCSL) was calculated. Hence, RFCSL expresses the percentage of 1-cm AMFcolonized segments to the total $1-\mathrm{cm}$ segments of all lateral roots; the number of total segments was $\approx 30$ per plant. Average colonization was calculated from the values of five plants.

Determination of free amino acids in plants. Sixteen weeks after AMF inoculation, plants were sampled and partitioned into ferns and storage roots from 10 plants, and all samples were frozen in liquid nitrogen. The samples for free amino acid analysis were collected from 10 plants as follows: ferns $(\approx 1 \mathrm{~cm}$ long from the base) and storage roots $(\approx 1 \mathrm{~cm}$ from the crown $)$. Samples were extracted in $0.2 \mathrm{~N}$ perchloric acid solution mixed with $0.25 \mu \mathrm{M}$ D,L-norleucine, and $\mathrm{pH}$ was adjusted to 4.0 with $\mathrm{KHCO}_{3}$, then the extracts were filtrated by a GL-chromatodisc (GL science Co., Ltd., Tokyo, Japan). Free amino acid concentrations (41 constituents) were measured using an automatic amino acid analyzer (JLC-500; JEOL Co., Ltd., Tokyo, Japan) using ninhydrin.

Fusarium oxysporum f. sp. asparagi culture with amino acids in vitro. The conidia of Foa (MAFF305556) grown on potato dextrose agar media was subcultured for 2 weeks on Czapec-Dox media (Ohata, 1995) containing $\mathrm{NaNO}_{3} 3 \mathrm{~g}, \mathrm{~K}_{2} \mathrm{HPO}_{4} 1 \mathrm{~g}, \mathrm{KCl} 0.5 \mathrm{~g}$, $\mathrm{MgSO}_{4} \cdot 7 \mathrm{H}_{2} \mathrm{O} \quad 0.5 \mathrm{~g}, \quad \mathrm{FeSO}_{4} \cdot 7 \mathrm{H}_{2} \mathrm{O} \quad 0.01 \mathrm{~g}$, sucrose $30 \mathrm{~g}$, agar $8 \mathrm{~g} \cdot \mathrm{L}^{-1}(\mathrm{pH}$ 5.8). The conidia were further subcultured $\left(10^{6}\right.$ con$\mathrm{idia} / \mathrm{mL}$ ) in liquid Czapec-Dox media with or without addition of filter-sterilized amino acids (arginine, GABA; 0.1, 1\%, w/v) at $25{ }^{\circ} \mathrm{C}$ in the dark for $7 \mathrm{~d}$ by shaken culture (100 rpm). Then, the density of conidia was investigated using a hemocytometer and calculated the propagation index of amino acid-added plots to amino acid-non-added plots. The average was calculated from eight replications.

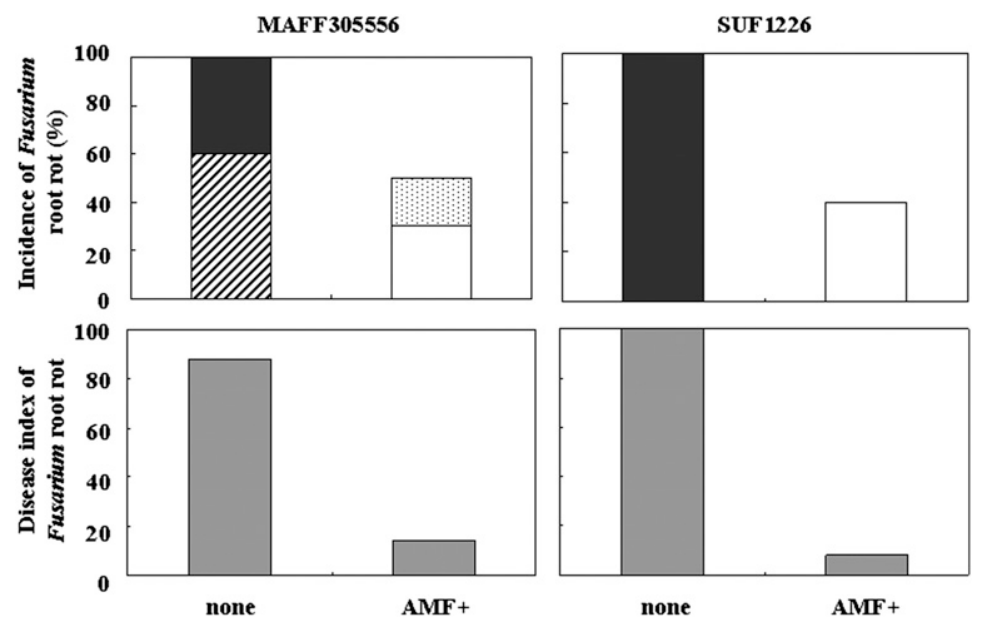

Fig. 2. Disease incidence and indices of fusarium root rot in mycorrhizal asparagus plants 10 weeks after Fusarium oxysporum f. sp. asparagi (MAFF305556, SUF1226) inoculation. None, AMF+, see Figure 1. Ratio of diseased storage roots. $\square, 0-20 ; \square, 20-40 ;$ 圆, 40-60; $\mathbb{Z}, 60-80 ; \square, 80-100$ (\%).

Statistical analysis. Mean values were separated by $t$ test for dry weight and free amino acid contents at $P \leq 0.05$. All analyses were performed using statistical analysis software (SSRI, Tokyo, Japan).

\section{Results}

Sixteen weeks after AMF inoculation, AMF plants had greater dry weight of ferns and roots than non-AMF plants (Fig. 1). AMF colonization was occurred successfully and reached up to $73.3 \% 16$ weeks after AMF inoculation (data not shown). As for evaluation of disease, non-AMF plants showed $100 \%$ incidence and high severity in the both isolates 10 weeks after Foa inoculation (Fig. 2); no disease symptom appeared in the plants without Foa inoculation (data not shown). However, AMF plants showed lower incidence and severity than non-AMF plants in the both isolates, and the disease indices of fusarium root rot reached more than 80 in non-AMF plants of the both isolates, whereas it was low at less than 20 in AMF plants.

Total amino acid contents in ferns and roots were higher in AMF plants than nonAMF plants 16 weeks after AMF inoculation (Fig. 3). Eight constituents of free amino acids such as glutamine arginine, aspartic acid, glutamic acid, citrulline, GABA, threonine, and histidine became higher in ferns of AMF plants than non-AMF (Fig. 4), whereas in roots of AMF plants, 16 constituents of amino acids such as asparagine, arginine, serine, glutamic acid, glutamine, GABA, aspartic acid, citrulline, valine, glycine, alanine, isoleucine, leucine, phenylalanine, histidine, and lysine increased (Fig. 5). In this case, arginine and GABA showed considerable increase in both ferns and roots in AMF plants. In the Foa culture by Czapec-Dox medium in vitro, arginine and GABA showed suppression effect on the index of Foa propagation in both $0.1 \%$ and $1 \%$ plots (Fig. 6).
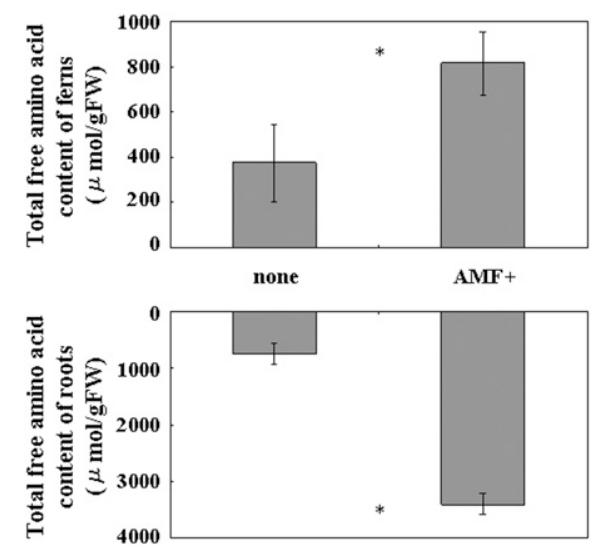

Fig. 3. Total free amino acid contents in mycorrhizal asparagus plants 16 weeks after AMF inoculation. None, AMF+; see Figure 1. Bars represent SES $(\mathrm{n}=10)$ * ${ }^{*}$ Significantly different between non-AMF and AMF plants $(t$ test, $P \leq$ $0.05) ;$ Ns $=$ non-significant.

\section{Discussion}

In the present study, increase in total amino acid content and several constituents occurred in mycorrhizal asparagus plants, although the effects varied between the plant parts. Sood (2003) reported the increases in glutamic acid, glycine, alanine, and leucine in mycorrhizal tomato seedlings, and Fattah and Mohamedin (2000) mentioned glutamic acid and serine increases in mycorrhizal sorghum plants. On the other hand, Baltruschat and Schonbeck (1975) demonstrated that in tobacco plants, an increase in both arginine and citrulline occurred in mycorrhizal plants. The results in this study have similar points as those reported for tomato, sorghum, and tobacco. Changes in GABA concentrations in mycorrhizal asparagus plants have not been reported by others; therefore, in this study, the increase of GABA concentrations in mycorrhizal asparagus plants is new information. Recently, GABA acts an important function in plant stress responses (Saito et al., 2008); in 

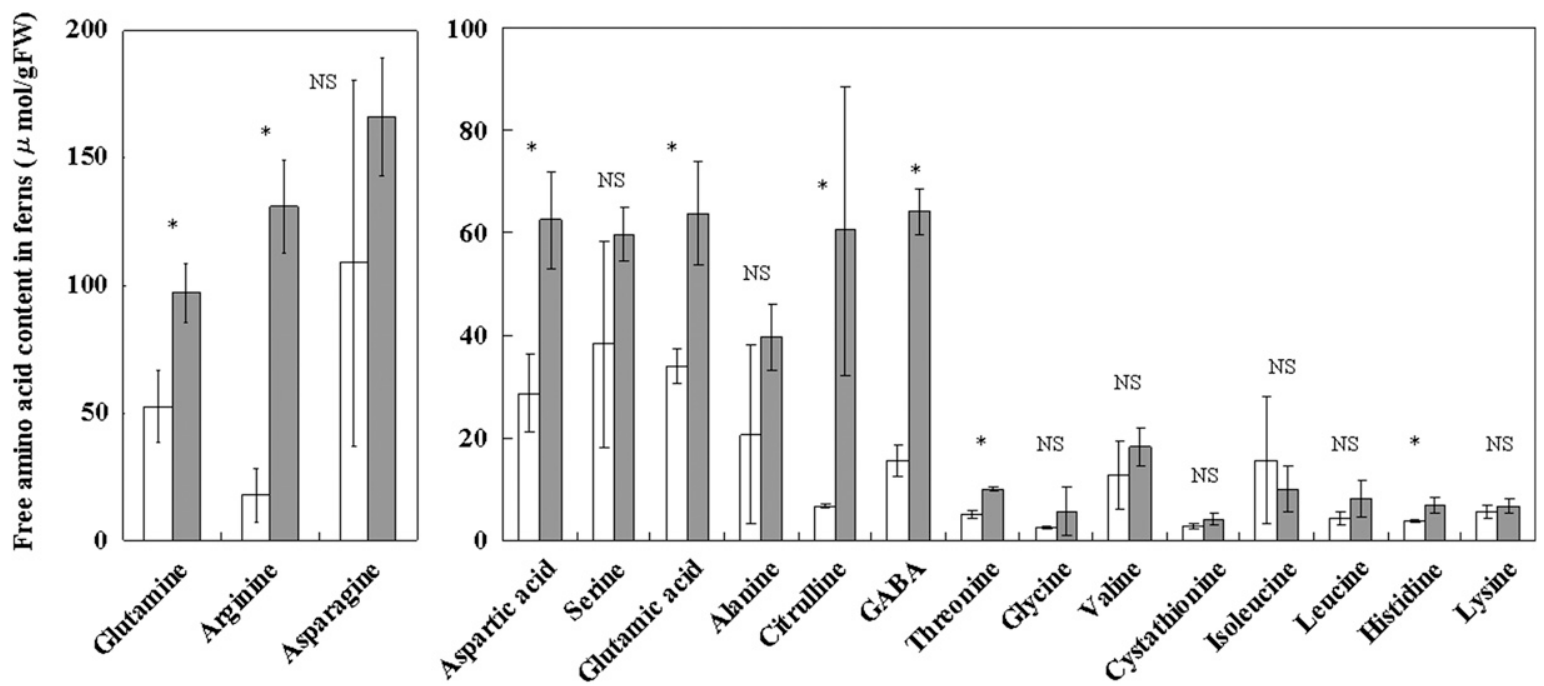

Fig. 4. Free amino acid contents in ferns 16 weeks after AMF inoculation. $\square$, non-AMF; $\square$, Glomus sp. R10. Bars represent SES ( $\mathrm{n}=10)$. * Significantly different between non-AMF and AMF plants ( $t$ test, $P \leq 0.05$ ); NS = non-significant. AMF = arbuscular mycorrhizal fungus.
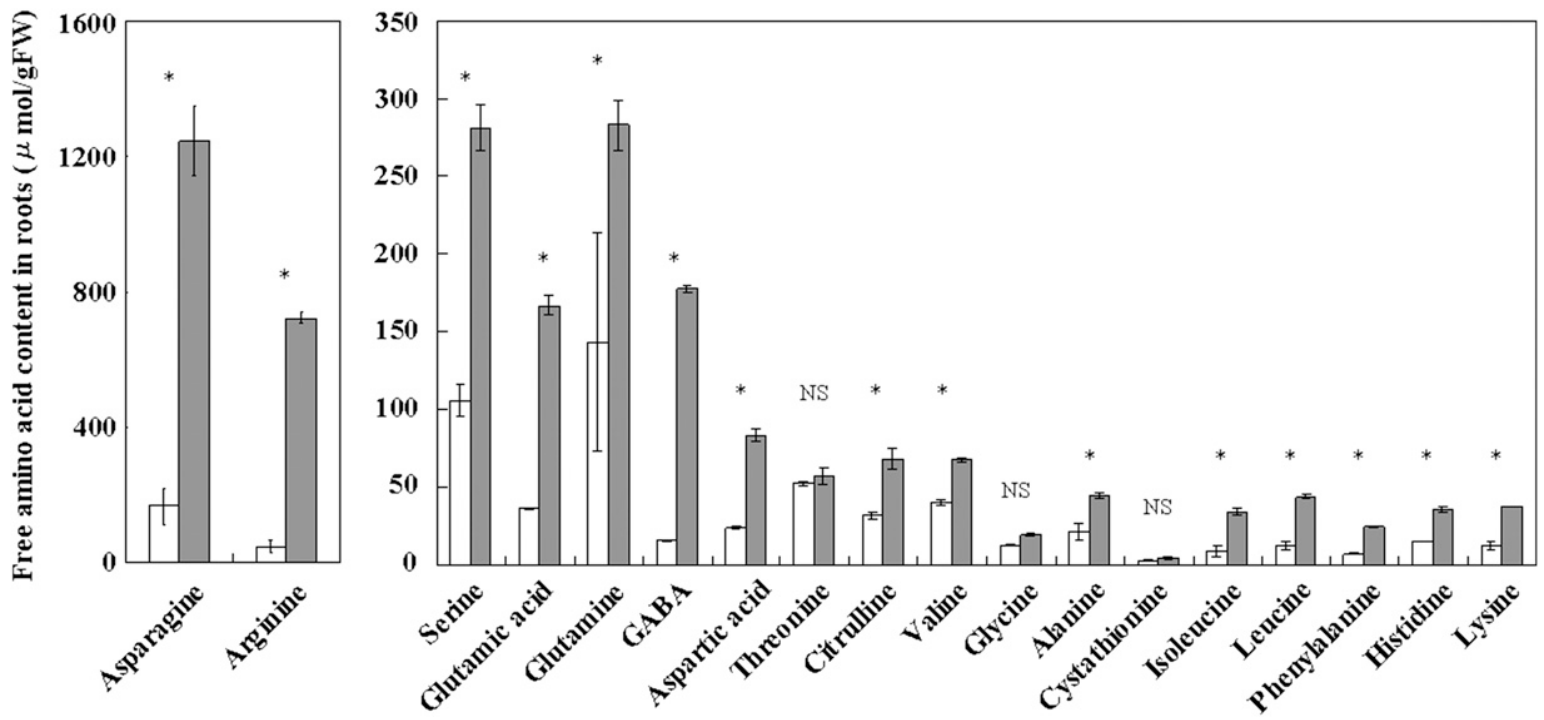

Fig. 5. Free amino acid contents in roots 16 weeks after AMF inoculation. $\square$, non-AMF; $\square$, Glomus sp. R10. Bars represent SES $(\mathrm{n}=10)$. *Significantly different between non-AMF and AMF plants ( $t$ test, $P \leq 0.05)$; NS = non-significant. AMF = arbuscular mycorrhizal fungus.

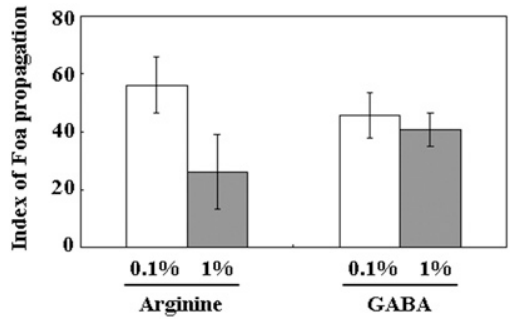

Fig. 6. Index of Foa (Fusarium oxysporum f. sp. asparagi; MAFF305556) propagation cultured by Czapec-Dox medium in vitro.

addition, the blood pressure-lowing effect of GABA in humans was reported (Inoue et al., 2003). From these findings, the increase of GABA in mycorrhizal asparagus plants of this study is interesting in the aspects of both disease tolerance and quality in harvested products.
Increase of free amino acid contents in mycorrhizal plants have been reported (Baltruschat and Schonbeck, 1975; Fattah and Mohamedin, 2000; Sood, 2003) and concentrations vary for several host-fungus combinations. Previous and this reports, thus, only one AMF species, so that it remains unclear whether a fungal difference in amino acid changes occurs in the same host. On the other hand, Fattah and Mohamedin (2000) mentioned that the degree of the increase in amino acids was correlated with the level of mycorrhizal colonization in the sorghumGlomus intraradices combination. Sutton (1973) demonstrated AMF colonization consisted of three phases: 1) a lag phase during which spore germination, germ tube growth, and initial penetration occur; 2) a rapid growth phase, coinciding with the development of external mycelium and spread of the fungus within the roots; and 3) a stable phase during which the proportion of infected roots to non-infected ones remains nearly constant. In our study, no significant difference was observed in colonization level between before and after (data not shown) Foa inoculation. However, in this experiment, amino acids were investigated only before Foa inoculation, because most of the non-AMF plants were withered after Foa inoculation, resulting in the difficulty of the amino acid analysis. Hence, it was difficult to estimate the fluctuation in colonization level and the relationship between free amino acid contents and colonization level.

In the present study, AMF promoted the growth of asparagus plants, and both the incidence and severity of symptoms in Foa were alleviated by pre-colonization with AMF. Baltruschat and Schonbeck (1975) demonstrated that in tobacco plants, an increase in both arginine and citrulline occurred in mycorrhizal plants, which inhibited the propagation of Thielaviopsis basicola. Starratt and 
Lazarovits (1999) reported low levels of the herbicide trifluralin induced resistance to fusarium wilt and elevated levels of free amino acids in melon seedlings. In this study, the increase in several free amino acids through mycorrhizal symbiosis in asparagus plants was confirmed, and arginine and GABA, which showed considerable increase in both ferns and roots in AMF plants, demonstrated suppression effect of Foa propagation. From these findings, tolerance to fusarium root rot in this study is closely associated with an increase in free amino acids as a direct factor to Foa propagation.

On the other hand, Pozo et al. (2002) reported that in tomato plants with a split root system, tolerance to Phytophthora parasitica appeared in both non-AMF-inoculated roots and inoculated roots in AMF plants, so that induced systemic disease tolerance was recognized. In this study, several free amino acids increased in ferns, where no colonization occurred. From these facts, we will estimate the induced systemic disease tolerance in mycorrhizal asparagus plants with a split root system, and further work is required to determine whether the changes in free amino acid contents have a direct or indirect relationship to the suppression of induced disease tolerance.

Our results suggest that AMF could induce tolerance to fusarium root rot in asparagus plants, and several free amino acids increased through the symbiosis. Some of the increased amino acids suppressed Foa propagation, leading to the disease tolerance as a physiological factor. Thus, control of fusarium diseases using AMF as a biocontrol agent seeks to develop a sustainable practice to manage the disease and improve plant health, thus contributing to an improvement in asparagus decline.

\section{Literature Cited}

Baltruschat, H. and F. Schonbeck. 1975. The influence of endotrophic mycorrhiza on the infestation of tobacco by Thielaviopsis basicola. Phytopathol. Z. 84:172-188.
Blok, W.J., M.J. Zwankhuizen, and G.J. Bollen. 1997. Biological control of Fusarium oxysporum f. sp. asparagi by applying non-pathogenic isolates of $F$. oxysporum. Biocont. Sci. Tech. 7:527-541.

Elmer, W.H. 2004. Combining nonpathogenic strains of Fusarium oxysporum with sodium chloride to suppress fusarium crown rot of asparagus in replanted fields. Plant Pathol. 53:751-758.

Fattah, G.M. and A.H. Mohamedin. 2000. Interactions between a vesicular-arbuscular mycorrhizal fungus (Glomus intraradices) and Streptomyces coelicolor and their effects on sorghum plants grown in soil amended with chitin of brawn scales. Biol. Fertil. Soils 32:401-409.

Hamel, C., V. Vujanovic, A. Nakano-Hylander, R. Jeannotte, and M. St-Arnaud. 2005. Factors associated with fusarium crown and root rot of asparagus outbreaks in Quebec. Phytopathology 95:867-873.

Inoue, K., T. Shirai, H. Ochiai, M. Kasao, K. Hayakawa, M. Kimura, and H. Sansawa. 2003. Blood-pressure-lowing effect of a novel fermented milk containing $\gamma$-aminobutyric acid (GABA) in mild hypertensives. Eur. J. Clin. Nutr. 57:490-495.

Knaflewski, M., P. Golinski, M. Kostecki, A. Waskiewicz, and Z. Weber. 2008. Mycotoxins and mycotoxin-producing fungi occurring in asparagus spears. Acta Hort. 776:183-189.

Lake, R.J., P.G. Falloon, and D.W.M. Cook. 1993. Replant problem and chemical components of asparagus roots. N. Z. J. Crop Hort. Sci. 21:53-58.

Marschner, H. and B. Dell. 1994. Nutrient uptake in mycorrhizal symbiosis. Plant Soil 159:89102.

Matsubara, Y., N. Hasegawa, and N. Ohba. 2003. Relation between fiber and pectic substance in root tissue and tolerance to fusarium root rot in asparagus plants infected with arbuscular mycorrhizal fungus. J. Jpn. Soc. Hort. Sci. 72:275280.

Miller, H.G., M. Ikawa, and L.C. Peirce. 1991. Caffeic acid identified as an inhibitory compound in asparagus root filtrate. HortScience 26:1525-1527.

Nahiyan, A.S.M., L.R. Boyer, P. Jeffries, and Y. Matsubara. 2011. PCR-SSCP analysis of Fusarium diversity in asparagus decline in Japan. Eur. J. Plant Pathol. 130:197-203.

Ohata, K. 1995. Cultivation of pathogenic fungi, p. 1-11. In: Ohata K, T. Araki, H. Kiso, A.
Kudo, and H. Takahashi (eds.). Methods for isolation, cultivation, inoculation of plant pathogens. Japan Plant Protection Association, Tokyo, Japan

Phillips, J.M. and D.S. Hayman. 1970. Improved procedures for clearing roots and staining parasitic and vesicular-arbuscular mycorrhizal fungi for rapid assessment of infection. Trans. Br. Mycol. Soc. 55:158-163.

Pozo, M.J., C. Cordier, E.D. Gaudot, J.M. Barea, and C.A. Aguilar. 2002. Localized versus systemic effect of arbuscular mycorrhizal fungi on defence responses to Phytophthora infection in tomato plants. J. Expt. Bot. 53:525-534.

Reid, T.C., M.K. Hausbeck, and K. Kizilkaya. 2001. Effects of sodium chloride on commercial asparagus and of alternative forms of chloride salt on fusarium crown and root rot. Plant Dis. 85:1271-1275.

Reid, T.C., M.K. Hausbeck, and K. Kizilkaya. 2002. Use of fungicides and biological controls in the suppression of fusarium crown and root rot of asparagus under green house and growth chamber conditions. Plant Dis. 86:493-498.

Rolin, D., P.E. Pfeffer, D.D. Douds, H.M. Farrell, and Y. Shachar. 2001. Arbuscular mycorrhizal symbiosis and phosphorus nutrition: Effects on amino acid production and turnover in leek. Symbiosis 30:1-14

Saito, T., C. Matsukura, M. Sugiyama, A. Watahiki, I. Ohshima, Y. Iijima, C. Konishi, T. Fujii, S. Inai, N. Fukuda, S. Nishimura, and H. Ezura. 2008. Screening for $\gamma$-aminobutyric acid (GABA)-rich tomato varieties. J. Jpn. Soc. Hort. Sci. 77:242-250.

Sood, S.G. 2003. Chemotactic response of plantgrowth-promoting bacteria towards roots of vesicular-arbuscular mycorrhizal tomato plants. Microb. Ecol. 45:219-227.

Starratt, A.N. and G. Lazarovits. 1999. Herbicideinduced disease resistance and associated increases in free amino acid levels in melon plants. Can. J. Plant Pathol. 21:33-36.

Sutton, J.C. 1973. Development of vesiculararbuscular mycorrhizae in crop plants. Can. J. Bot. 51:2487-2493.

Wong, J.Y. and P. Jeffries. 2006. Diversity of pathogenic fusarium populations associated with asparagus roots in decline soils in Spain and the UK. Plant Pathol. 55:331-342.

Yong, C.C. 1984. Autointoxication in root exudates of Asparagus officinalis L. Plant Soil 82:247-253. 